dans ce groupe, quelques-uns qui soient inoffensifs; car ils ne peuvent pas, en tout cas, être de quelque utilité, et la reconnaissance facile des organismes étrangers fait $q u$ 'on peut rapidement savoir s'il est nécessaire de s'en débarrasser, tandis qu'une évaluation de la nocivité de chacune des différentes sortes exigerait des recherches sans fin. Nous devons done nous contenter de nous tenir au point de vue suivant : les organismes qui ne doivent pas être dans le beurre doivent en être écartés.

\title{
BIBLIOGRAPHIE.
}

[1] Hesse. Molkerei-Ztg., Hildesheim, 1912, no 6 (v. Orla -Jensen : Bactériologie laitière, p. 139).

[2] C. O. JENSEN. 22e rapport du Laboratoire d'Essais, 1891.

[3] Orta-Jensen. Mcelkeritidende, 1907, p. 943.

[4] Orla-Jensen. Rev. Gén. du Lait, 1910, p. 409.

[5] OrLa-Jensen. Annuaire agricole de Suisse, 1901, p. 329.

[6] Sтовсн. Ugeskrift for Landmoend, 1875, 11, p. 563.

[7] Sтовсн. 36' rapport du Laboratoire d'Essais, 1897.

[8] Rahn et Boysen. Milchw. Forsch., 1929, vol. 7, p. 214.

[9] Boysen. Milchw. Forsch., 1927, vol, 4, p. 221.

[10] Chr. H. IBSEn. 106 rapport du Laboratoire d'Essais, 1921.

[11] BurRI. Congrès international de Laiterie, 1928, p. 690.

[12] Dyrenfurth et Richter. Milchw. Forsch., 1929, vol. 8, p. 462.

\section{CONTRIBUTION A L'ÉTUDE DE LA LACTATION HUMAINE}

par

Mme C. VINCENT et

Chef des travaux

M. J. VIAL

Assistant

du Laboratoire de Physiologie de la Faculté de Médecine de Lyon.

(Suite.)

c) QUANTITÉS COMPARÉS FOURNIES PAR LES DIFFÉRENTES MULSIONS, AU COURS D'UNE MEME JOURNEE. - On ne peut comparer légitimement des mulsions que si elles sont complètes. Par conséquent, ne pourront être utilisées dans ce but que les mulsions recueillies manuellement. On peut utiliser encore les chiffres fournis par le repas pesé du nourrisson, augmenté s'il y a lieu de la quantité fournie par la traite manuelle, toujours pratiquée pour vérifier l'épuisement complet de la glande.

Il importe naturellement, en outre, que les mulsions à comparer soient bien faites à des heures identiques, afin d'éviter tout phénomène de rétention qui fausserait les résultats. 
Dans ces conditions, on peut noter, d'une façon générale, ce qui suit :

Dans les cas où il y a de très nombreuses mulsions ( 6 en moyenne par 24 heures : $8,11,14,18,21,24$ h.), la récolte la plus importante est généralement celle du matin, mais elle n'est pas beaucoup plus abondante que les autres (cette abondance est, d'ailleurs, fonction de l'heure à laquelle a eu lieu la dernière tétée de la journée précédente). Les récoltes du milieu de la journée sont très variables; celle de $14 \mathrm{~h}$. est un peu moins importante peut-être que celles de $11 \mathrm{~h}$. et de $17 \mathrm{~h}$. Le plus souvent, la plus faible récolte de la journée est celle de 21 h., tandis qu'au contraire, celle de $24 \mathrm{~h}$. donne une quantité sensiblement plus élevée que toutes les autres traites, à l'exception de celle du matin ( $C f$. courbe $\mathrm{L}$ ).

Dans le cas d'une lactation régulière, mais moins intense (3 mulsions par jour : $8,14,24$ h.), on peut faire les mêmes remarques, avec plus de netteté encore. De plus, les exceptions à la règle générale sont moins nombreuses. La récolte du matin reste, presque toujours, et de beaucoup, la plus abondante, et elle représente depuis 1,5 fois jusqu'à 2,5 fois la moyenne des deux autres. La récolte de $14 \mathrm{~h}$. est dans l'ensemble un peu plus faible que celle de minuit, dont elle se rapproche toutefois assez sensiblement ( $C f$. courbe A).

d) QUANTITÉS COMPARÉES FOURNIES PAR LES MULSIONS HOMOloques, Au cours des journeEs successives. - Ces traites homologues (e'est-à-dire obtenues à la même heure) présentent, elles aussi, des caractères de variabilité très marqués.

Par exemple, si l'on considère la première mulsion de la journée, celle-ci variera souvent d'un jour à l'autre du simple au double, parfois plus. Il en sera de même pour les autres traites.

Néanmoins, les variations des unes et des autres ne détruisent pas dans l'ensemble les rapports d'importance des traites quotidiennes entre elles, tels que nous les avons notés au paragraphe précédent.

e) COMPARAISON DE LA QUANTITÉ SÉcRÉtEe PAR CHACUN dES seıns. - Nous avons observé ici, d'une façon précise, un fait bien connu des nourrices. La plupart d'entre elles disent couramment que l'un de leurs seins donne une sécrétion plus abondante que l'autre, ce qui se vérifie presque toujours.

Si on pratique, comme on le doit, à une heure donnée, séparément et à fond, la mulsion de chaque sein, on constate que :

10 Le plus souvent, l'un d'eux (le droit ou le gauche) donne, d'une façon très constante; une quantité supérieure à celle de l'autre. Notons, en passant, que le sein le plus productif n'est pas toujours le 


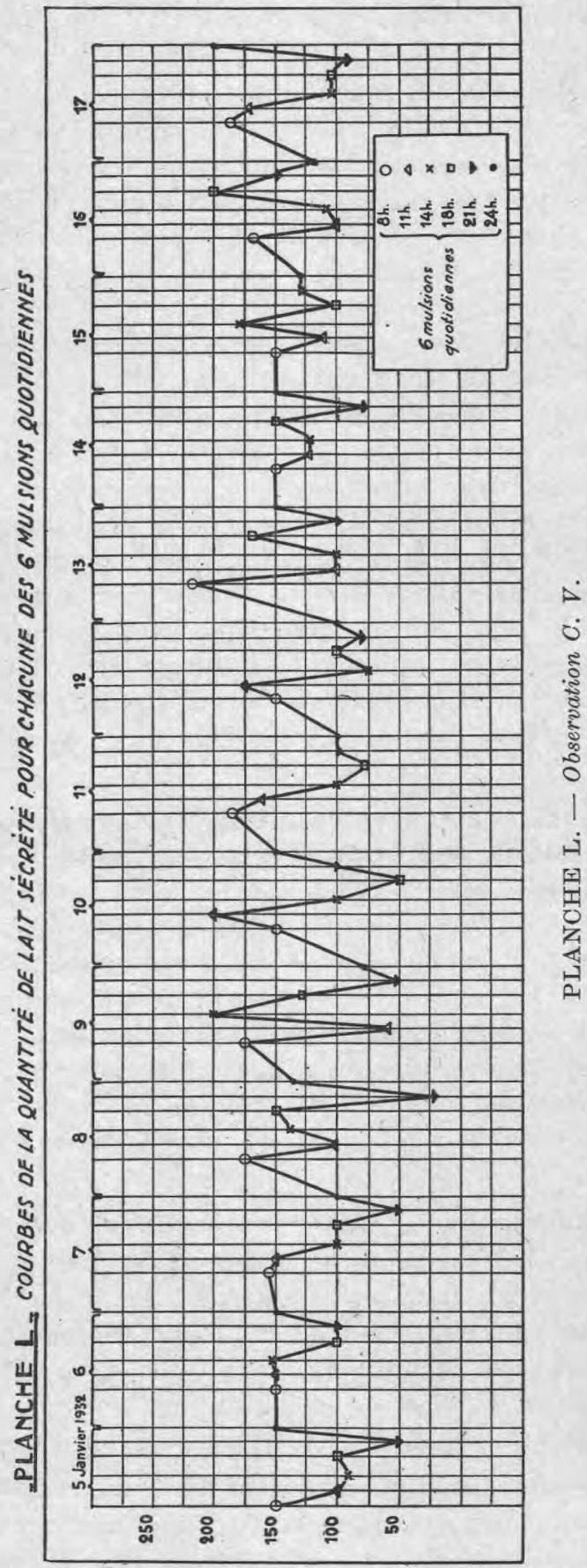




\begin{tabular}{|c|c|c|c|c|c|c|c|c|c|}
\hline Date & $\begin{array}{l}\text { Heure de } \\
\text { la mulsion }\end{array}$ & $\begin{array}{c}\text { No de } \\
\text { la mulsion }\end{array}$ & $\begin{array}{l}\text { Quantité } \\
\text { de lait } \\
\text { Sein D. }\end{array}$ & $\begin{array}{c}\text { Quantité } \\
\text { de lait } \\
\text { Sein G. }\end{array}$ & $\begin{array}{c}\text { Taux } \\
\text { butyreux } \\
\text { D. }\end{array}$ & $\begin{array}{c}\text { Taux } \\
\text { butyreux } \\
\text { G. }\end{array}$ & $\begin{array}{c}\text { Poids de } \\
\text { matières } \\
\text { grasses } \\
\text { D. }\end{array}$ & $\begin{array}{c}\text { Poids de } \\
\text { matières } \\
\text { grasses } \\
\text { G. }\end{array}$ & $\begin{array}{c}\text { Observa } \\
\text { tions }\end{array}$ \\
\hline 23 & $\begin{array}{r}8 \\
14 \\
24\end{array}$ & $\begin{array}{r}I \\
I I \\
I I I\end{array}$ & $\begin{array}{r}105 \\
70 \\
60\end{array}$ & $\begin{array}{l}80 \\
40 \\
45\end{array}$ & $\begin{array}{l}44 \\
59,8 \\
34,9\end{array}$ & $\begin{array}{l}52,8 \\
52,7 \\
26,7\end{array}$ & $\begin{array}{l}4,62 \\
4,19 \\
2,09\end{array}$ & $\begin{array}{l}4,22 \\
2,11 \\
1,20\end{array}$ & \\
\hline 24 & $\begin{array}{r}8 \\
14 \\
23\end{array}$ & $\begin{array}{r}\text { I } \\
\text { II } \\
\text { III }\end{array}$ & $\begin{array}{r}150 \\
83 \\
80\end{array}$ & $\begin{array}{r}120 \\
57 \\
48\end{array}$ & $\begin{array}{l}42,9 \\
75,1 \\
34,8\end{array}$ & $\begin{array}{l}45,6 \\
71 \\
31,5\end{array}$ & $\begin{array}{l}6,43 \\
6,23 \\
2,78\end{array}$ & $\begin{array}{l}5,47 \\
4,05 \\
1,51\end{array}$ & \\
\hline 25 & $\begin{array}{r}8 \\
14 \\
24\end{array}$ & $\begin{array}{r}\text { I } \\
\text { II } \\
\text { III }\end{array}$ & $\begin{array}{r}125 \\
34 \\
68\end{array}$ & $\begin{array}{r}100 \\
21 \\
52\end{array}$ & $\begin{array}{l}43,3 \\
38,5 \\
27\end{array}$ & $\begin{array}{l}37 \\
43,8 \\
29\end{array}$ & $\begin{array}{l}5,41 \\
1,31 \\
1,84\end{array}$ & $\begin{array}{l}3,7 \\
0,92 \\
1,51\end{array}$ & \\
\hline 26 & $\begin{array}{r}8 \\
14 \\
24\end{array}$ & $\begin{array}{r}\text { I } \\
\text { II } \\
\text { III }\end{array}$ & $\begin{array}{r}145 \\
80 \\
95\end{array}$ & $\begin{array}{r}125 \\
55 \\
55\end{array}$ & $\begin{array}{l}37,9 \\
66,3 \\
34,1\end{array}$ & $\begin{array}{l}45,7 \\
62,9 \\
30,9\end{array}$ & $\begin{array}{l}5,50 \\
5,30 \\
3,24\end{array}$ & $\begin{array}{l}5,71 \\
3,46 \\
1,70\end{array}$ & \\
\hline 27 & $\begin{array}{r}8 \\
14 \\
24\end{array}$ & $\begin{array}{r}\text { I } \\
\text { II } \\
\text { III }\end{array}$ & $\begin{array}{r}115 \\
33 \\
88\end{array}$ & $\begin{array}{l}80 \\
27 \\
62\end{array}$ & $\begin{array}{l}55,2 \\
51,7 \\
32,8\end{array}$ & $\begin{array}{l}42,3 \\
49,2 \\
31,6\end{array}$ & $\begin{array}{l}6,35 \\
1,70 \\
2,89\end{array}$ & $\begin{array}{l}3,38 \\
1,33 \\
1,96\end{array}$ & . \\
\hline 28 & $\begin{array}{r}8 \\
14 \\
23\end{array}$ & $\begin{array}{r}\text { I } \\
\text { II } \\
\text { III }\end{array}$ & $\begin{array}{r}135 \\
74 \\
112\end{array}$ & $\begin{array}{r}100 \\
63 \\
63\end{array}$ & $\begin{array}{l}45,8 \\
71 \\
45,2\end{array}$ & $\begin{array}{l}43,9 \\
69,5 \\
40,4\end{array}$ & $\begin{array}{l}6,18 \\
5,26 \\
5,06\end{array}$ & $\begin{array}{l}4,39 \\
4,38 \\
2,55\end{array}$ & \\
\hline 29 & $\begin{array}{r}8 \\
15 \\
23\end{array}$ & $\begin{array}{r}\text { I } \\
\text { II } \\
\text { III }\end{array}$ & $\begin{array}{r}105 \\
65 \\
80\end{array}$ & $\begin{array}{l}65 \\
50 \\
60\end{array}$ & $\begin{array}{l}58,1 \\
46,3 \\
35,5\end{array}$ & $\begin{array}{l}41,6 \\
44,7 \\
37,4\end{array}$ & $\begin{array}{l}6,10 \\
3,01 \\
2,84\end{array}$ & $\begin{array}{l}2,70 \\
2,23 \\
2,24\end{array}$ & \\
\hline 30 & $\begin{array}{r}8 \\
14 \\
24\end{array}$ & $\begin{array}{r}I \\
I I \\
I I I\end{array}$ & $\begin{array}{r}125 \\
33 \\
125\end{array}$ & $\begin{array}{r}105 \\
27 \\
75\end{array}$ & $\begin{array}{l}45 \\
54,8 \\
32,1\end{array}$ & $\begin{array}{l}50 \\
57,5 \\
32,3\end{array}$ & $\begin{array}{l}5,62 \\
1,81 \\
4,01\end{array}$ & $\begin{array}{l}5,25 \\
1,55 \\
2,42\end{array}$ & \\
\hline 31 & $\begin{array}{r}8 \\
14 \\
23\end{array}$ & $\begin{array}{r}\text { I } \\
\text { II } \\
\text { III }\end{array}$ & $\begin{array}{r}125 \\
40 \\
120\end{array}$ & $\begin{array}{r}100 \\
30 \\
60\end{array}$ & $\begin{array}{l}52,9 \\
56,7 \\
38,6\end{array}$ & $\begin{array}{l}47,7 \\
59,9 \\
35,3\end{array}$ & $\begin{array}{l}6,61 \\
2,27 \\
4,63\end{array}$ & $\begin{array}{l}4,77 \\
1,80 \\
2,12\end{array}$ & \\
\hline$l_{\text {er }}$ & $\begin{array}{r}8 \\
14 \\
24\end{array}$ & $\begin{array}{r}I \\
\text { II } \\
\text { III }\end{array}$ & $\begin{array}{l}85 \\
88 \\
90\end{array}$ & $\begin{array}{l}75 \\
77 \\
55\end{array}$ & $\begin{array}{l}41 \\
53,2 \\
23,4\end{array}$ & $\begin{array}{l}35 \\
37 \\
24,9\end{array}$ & $\begin{array}{l}3,48 \\
4,68 \\
2,11\end{array}$ & $\begin{array}{l}2,52 \\
2,85 \\
1,37\end{array}$ & \\
\hline
\end{tabular}


plus volumineux à l'inspection (l'asymétrie mammaire est, en effet, classique). Cette asymétrie est d'ailleurs fonction autant des couches adipeuses superficielles que de la masse même de la glande.

$2^{\circ} \mathrm{L}$ 'écart de quantité sécrétée entre le sein droit et le sein gauche est variable selon les femmes, mais assez constant pour chacune d'elles.

$3^{\circ}$ Cet écart atteint souvent $30 \%$ et plus.

$4^{\circ}$ En tout cas, en suivant les courbes des mulsions successives, on voit que les quantités sécrétées par l'un et l'autre sein varient d'une façon remarquablement parallèle. Quand un sein donne beaucoup, l'autre donne beaucoup aussi, et vice versa ( $C f$. courbe $\mathrm{G}$ ).

\section{- PLANCHE G - COURBES DES QUANTITÉS DE LAIT SÉCRETÉES \\ A CHAQUE MULSION PAR CHACUN DES DEUX SEINS}

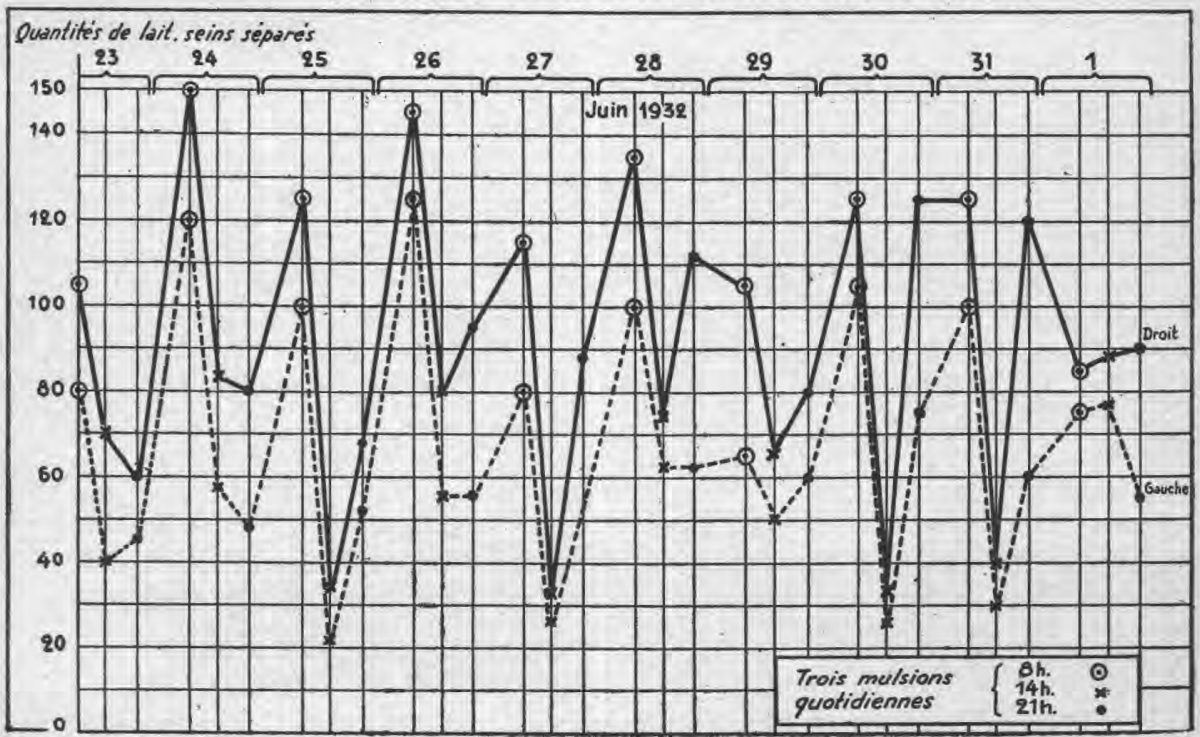

PLANCHE G. - Observation $C . V$.

L'activité fonctionnelle des deux seins se développe done toujours dans le même sens, quel que soit ce dernier.

$5^{\circ}$ Ces différences de quantité tiennent aux possibilités sécrétoires de la glande, bien que les nourrices l'attribuent, en général, à une préférence du nourrisson.

En effet, quand la totalité de la sécrétion est obtenue par mulsions, sans que l'enfant tète au sein, on voit persister indéfiniment des différences quantitatives de même ordre, au profit de l'un des seins.

f) VARIATION DU DEBIT AU COURS DE LA MULSION. - 1 . Au 
début d'une tétée, si l'on observe les déglutitions de l'enfant, on peut aisément se rendre compte que le débit du lait fourni par le sein s'accroît peu à peu, mais modérément.

Au bout d'un temps variant de quelques secondes à quelques minutes, on note souvent une période de ralentissement du débit ou même d'arrêt. Ce phénomène est tel qu'il provoque parfois les cris de l'enfant. Après quelques nouvelles minutes, la "montée de lait " a lieu, comme le savent toutes les nourrices, et le débit devient brusquement très abondant, au point de gêner l'enfant, qui alors tousse et ne réussit pas à tout avaler.

Au moment où se produit la "montée de lait" au sein tété par l'enfant, la nourrice éprouve une sensation particulière au niveau des deux seins, simultanément ou à quelques secondes d'intervalle chez certaines femmes. On note souvent alors, au sein qui n'est pas tété, un écoulement spontané du lait, lequel tombe goutte à goutte. Cet écoulement atteint et dépasse $5 \mathrm{~cm}^{3}$; de légers pincements du mamelon le font généralement cesser.

Nous n'avons rappelé ces détails, en les précisant, que pour signaler l'existence des mêmes phénomènes au cours de la mulsion pratiquée au tire-lait, ou surtout par le procédé manuel.

Dans ce dernier cas, les pressions digitales sur l'aréole trouvent au début celle-ci facilement dépressible, ainsi que le mamelon. L'écoulement se fait alors goutte à goutte ou par deux ou trois jets de faible pression.

A la phase de ralentissement ou d'arrêt, l'aréole et le mamelon restent très mous sous le doigt, et l'écoulement se réduit à des gouttes espacées ou cesse tout à fait. Des tentatives pour obtenir quelque chose de l'autre sein sont aussi infructueuses. La durée de cette dépression du débit varie selon les mulsions et selon les sujets. Elle manque rarement, mais est plus ou moins nette et prolongée. Elle est maxima chez les femmes impressionnables, et, - on peut le dire, - chez les plus entraînées aussi, à l'oceasion de la moindre contrariété. Elle peut, parfois, atteindre un quart d'heure, ou plus. Nous aurons à y revenir en signalant plus loin quelques influences psychiques susceptibles de modifier le débit du lait. Il faut alors continuer avec douceur et patience les pressions rythmées de la mulsion.

$\mathrm{Au}$ moment de la "montée laiteuse ", tout change en quelques secondes, parfois très brusquement; les doigts, posés sur l'aréole, perçoivent une rénitence toute spéciale de celle-ci, ainsi que du mamelon qui est en érection; le lait s'échappe avec une pression 5 ou 10 fois plus forte, en une quinzaine de jets qui sont soutenus durant 1 ou 2 secondes; la zone rétro-mamelonnaire vidée se tend de nouveau aussitôt sous le doigt, dont la pression déterminera 
l'évacuation de nouveaux jets. La quantité de lait recueillie à chaque mouvement rythmé peut atteindre 1 à $2 \mathrm{~cm}^{3}$.

La plus grande partie du lait contenu dans la glande est évacuée pendant cette période. Quand la glande est presque épuisée, le nombre des jets, leur durée et leur pression diminuent, ainsi que la consistance érectile de l'organe. Ce n'est bientôt plus qu'un goutteà-goutte déeroissant. Le rythme de la traite doit être alors ralenti, coupé de pauses, jusqu'à ce que l'on n'obtienne plus de lait. La rénitence a disparu.

Dans les conditions habituelles, si elle est tranquille, une femme bien entraînée aux manœuvres de mulsion peut effectuer facilement une traite complète de $100 \mathrm{~cm}^{3}$ environ en 5 minutes, le rythme. des pressions digitales maintenu environ à 70 par minute.

Le premier sein (droit ou gauche) étant vidé, on commence à tirer l'autre : généralement le débit du lait est aussitôt rapide et abondant. On ne note ni début progressif ni suspension, même partielle, et tout se passe comme pour le premier sein, depuis le moment de la "montée laiteuse ".

Ces phénomènes, retrouvés chez la femme avec la plus grande netteté, sont analogues à ceux qui existent chez les femelles des animaux domestiques : vache, mouton, chèvre, etc. Là aussi, se succèdent trois phases : $1^{\circ}$ mise en train progressive au début de la traite ; $2^{\circ}$ diminution du débit ou arrêt momentané ; $3^{\circ}$ montée laiteuse constituant l'exerétion proprement dite des mamelles.

On peut d'ailleurs "voir " très nettement ces phénomènes dans les courbes $\mathrm{X}$ et $\mathrm{Y}$.

Les expériences, qui ont permis de les établir, ont été pratiquées avec traite manuelle, poussée à fond, au rythme régulier de 70 pressions par minute.

Le lait sécrété pendant chacune des minutes était recueilli dans une série d'éprouvettes graduées et numérotées; on notait la quantité, et l'échantillon était affecté aux dosages.

Sur les courbes ainsi obtenues.

Si l'on envisage l'accroissement progressif de la quantité de lait sécrétée (courbe $X$, ligne supérieure), on voit nettement un point d'inflexion (phase d'inhibition), suivi aussitôt d'une brusque ascension (montée laiteuse).

Si l'on envisage les quantités sécrétées par minute (courbe $\mathrm{Y}$, ligne supérieure) on constate encore plus nettement ces phénomènes.

Après une période de début pendant laquelle les quantitésminute varient peu, on voit un très brusque fléchissement (phase d'inhibition), suivi d'une ascension brusque (montée laiteuse). A la fin de la mulsion, les quantités-minute décroissent progressivement.

Ces phénomènes que nous venons d'exposer et de vérifier en les 
mesurant, sont bien nets, mais leur cause paraît beaucoup plus obscure. Tant au point de $\nabla u e$ du débit qu'au point de vue des changements de consistance de l'organe, ils sont, - nous l'avons dit, - bilatéraux et simultanés. On est en droit de penser qu'ils sont d'ordre réflexe, d'ailleurs assez complexe.

Dans le cas particulier, se sont les manceuvres de mulsion au niveau de l'aréole qui déclenchent les réflexes.

Après avoir observé ces phénomènes des milliers de fois, nous avons voulu pratiquer l'enregistrement graphique, tant au cours de la traite successive des seins qu'au cours de leur traite simultanée.

II. Enregistrement graphique de la mulsion, successivement dans l'un et l'autre sein. - a) Dispositif expérimental (Cf. schéma). - Le lait, dont on se propose d'enregistrer le débit, est recueilli dans un entonnoir placé à 4 ou $5 \mathrm{~cm}$. au-dessous du mamelon.

Cet entonnoir est relié à un tube en verre de $0 \mathrm{~cm}$. 5 de diamètre et de $0 \mathrm{~m} .75$ de longueur, fixé à un support qui le maintient selon une pente de $45^{\circ}$.

Au-dessous de l'extrémité de ce tube, et sans contact avec lui (pour éviter que des mouvements de la nourrice soient transmis à tout le dispositif), se trouve un second entonnoir, où vient tomber le lait que laisse écouler le tube.

Ce second entonnoir est muni à son extrémité inférieure d'un tube de caoutchouc, lequel, terminé en bec de flûte, vient s'appliquer sur le bord de la paroi interne d'une éprouvette graduée de $200 \mathrm{~cm}$. et d'un diamètre de $3 \mathrm{~cm}$.

L'intérieur de cette éprouvette est muni d'un flotteur, fait d'un bouchon de liège soigneusement paraffiné, de $2 \mathrm{~cm}$. 5 de diamètre. Dans ce bouchon, est planté verticalement un fil d'aluminium rigide, auquel est fixé perpendiculairement, avec de la cire, un style inscripteur.

Un guide métallique placé à la partie supérieure de l'éprouvette a un double but :

1. Maintenir le tube adducteur de lait au contact de la paroi ;

$2^{\circ}$ S'opposer aux mouvements de latéralité de l'axe portant le style inscripteur.

Enfin, ce dernier vient au contact d'un enregistreur pourvu d'un mouvement d'horlogerie à vitesse réglable (modèle utilisé à l'Université Harward) et il y est maintenu par un fil à plomb très léger.

Dans la pratique, la nourrice est assise confortablement. Elle tient elle-même au-dessous et en avant de son sein le premier entonnoir récepteur. La personne qui pratique la mulsion se place 
debout derrière la nourrice, après avoir mis en marche l'appareil enregistreur. Elle prend aussitôt l'aréole entre le pouce et l'index et effectue les pressions rythmées avec une absolue régularité, à la moyenne courante de 70 par minute, moyenne qui sera respectée jusqu'à complet épuisement de la glande et jusqu'à la fin de l'expérience.

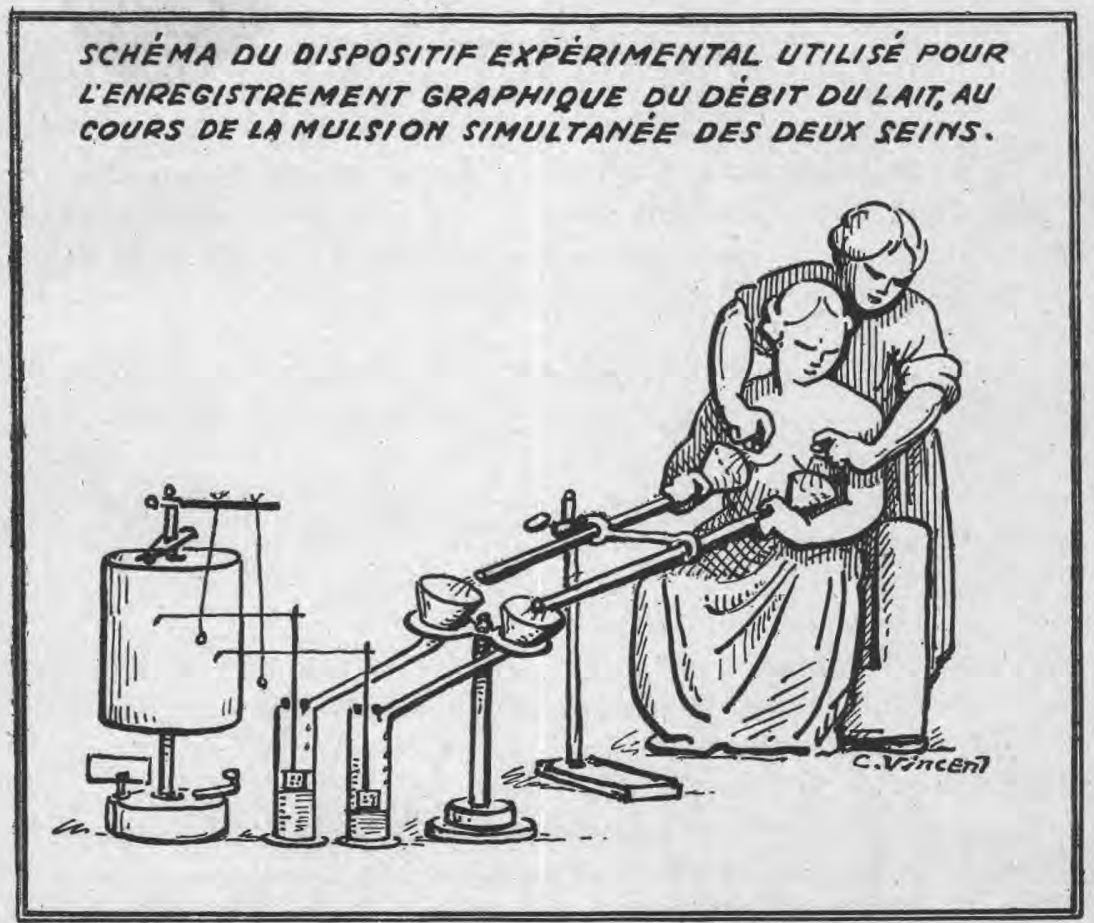

Le lait, tombant dans le premier entonnoir, chemine goutte à goutte le long du tube de verre, tombe dans le second entonnoir et vient glisser par le tube de caoutchouc le long de la paroi de l'éprouvette. Le niveau monte peu à peu, soulevant avec lui le flotteur inscripteur.

Aussitôt terminée la mulsion du premier sein, on décale simplement le eylindre enregistreur pour isoler les tracés, on transporte le premier entonnoir sous l'autre sein et on recommence l'opération.

b) Interprétation des tracés obtenus. - Le graphique porte en abcisses les temps et en ordonnées les quantités de lait.

La mulsion du premier sein (gauche) donne une courbe complexe, où nous retrouvons 4 périodes (Cf. tracé I) :

$1^{\circ}$ La ligne est d'abord oblique ascendante à $27^{\circ}$, correspondant à la phase de début de l'écoulement du lait, signalé plus haut. 
$2^{\circ}$ Bientôt, elle se rapproche de l'horizontale (phase d'inhibition).

$3^{\circ}$ Brusquement, elle devient oblique ascendante à $50^{\circ}$ (phase de la montée laiteuse).

$4^{\circ}$ La montée s'atténue légèrement, en restant régulièrement ascendante (phase terminale de la mulsion, traduisant l'épuisement de la glande).

La mulsion du second sein (droit), qui suit pour ainsi dire immédiatement, donne une courbe d'un aspect sensiblement différent, plus simple :

$1^{\circ}$ Les phases 1 et 2 manquent.

$2^{\circ}$ Le tracé montre dès le début une oblique ascendante très nette à $48^{\circ}$.

$3^{\circ}$ Celle-ci s'abaisse légèrement, restant toujours ascendante jusqu'à terminaison de la mulsion.

Done, alors que pour la première mulsion il y a eu une phase de début suivie d'une inhibition temporaire, il n'y a rien eu de semblable lors de la deuxième mulsion.

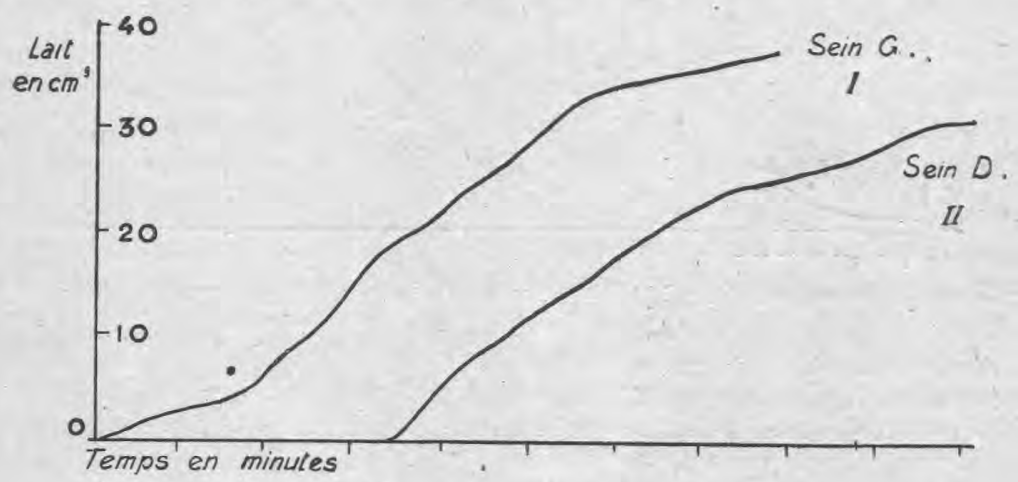

TRACÉ I. - Observation MHe $M$. $R$.

Enregistrement graphique du débit du lait au cours de la mulsion successive des deux seins.

(Pressions au rythme de 70 par minute.)

La phase d'inhibition précédant la montée laiteuse est indiquée par un point. Elle n'existe que sur le tracé du sein tiré le premier.

En effet, si l'on réfléchit que l'inhibition et la montée laiteuse sont déclanchées par voie réflexe simultanément dans les deux seins, on comprend que l'inscription du premier phénomène n'ait lieu qu'au cours de la première mulsion enregistrée. Au moment où commence l'inscription de la deuxième mulsion, l'inhibition est déjà terminée et la montée laiteuse est chose acquise. Ce tracé indiquera done d'emblée un débit maximum.

Cette interprétation est légitimée par les résultats exposés au paragraphe suivant. 
c) Enregistrement graphique de la mulsion simultanée des deux seins, - $a$ ) Nous avons utilisé l'appareil décrit plus haut, mais en doublant toute l'installation d'une façon rigoureusement semblable. Les éprouvettes étaient disposées de telle sorte que les. deux stylets inscripteurs, légèrement décalés, venaient marquer simultanément leurs tracés sur un même eylindre enregistreur (Cf. schéma).

La nourrice, installée comme ci-dessus, tenait un entonnoir sous chaque sein avec la main correspondante.

L'opérateur, placé derrière elle, pratiquait, une main à chaque sein, les mancuvres rythmées de mulsion, s'appliquant à ce que celles-ci soient bien simultanées, de même fréquence et de même force.

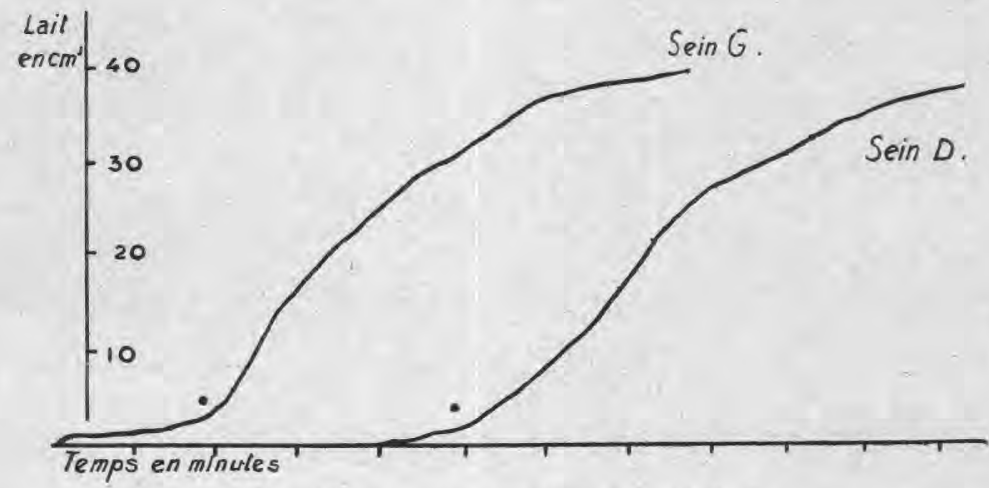

TRACÉ Tl. - Observation M11e M. L. R.

Enregistrement graphique du débit du lait au cours de la mulsion simultanée des deux seins.

(Pressions au rythme de 70 par minute.)

La phase d'inhibition précédant la montée laiteuse est indiquée par un point. Elle existe sur le tracé des deux seins.

b) Interprétation des tracés (tracé II). - Contrairement à ce que nous avons vu dans le cas des mulsions successives, on voit ici, sur les deux tracés concomitants, s'inscrire simultanément :

$1^{\circ}$ Une ligne très faiblement ascendante au début;

$2^{\circ}$ Un plateau horizontal correspondant à la phase d'inhibition;

$3^{\circ}$ Une ligne brusquement ascendante (la montée laiteuse);

$4^{\circ}$ Enfin, une ligne ascendante, plus atténuée à la fin de la mulsion.

Ces tracés rendent évidentes la simultanéité et la bilatéralité des différentes variations du débit, au cours de la mulsion.

Ils soulignent aussi l'importance des actions réflexes dans l'excrétion lactée. 